\title{
$\delta$-CS: A Direct-Band-Gap Semiconductor Combining Auxeticity, Ferroelasticity, and Potential for High-Efficiency Solar Cells
}

\author{
Minglei Sun and Udo Schwingenschlögl* \\ Physical Science and Engineering Division (PSE), King Abdullah University of Science and Technology (KAUST), \\ Thuwal 23955-6900, Saudi Arabia
}

(Received 27 July 2020; accepted 25 August 2020; published 9 October 2020)

\begin{abstract}
We propose a two-dimensional material, $\delta$-CS, and show that it is a direct-band-gap semiconductor with strong absorption of solar radiation. Power conversion efficiencies of $7.0 \%$ to $20.1 \%$ are predicted for solar cells with $\delta$-CS as a donor and a transition metal dichalcogenide as an acceptor. $\delta$-CS also excels in terms of having an exceptionally large negative in-plane Poisson's ratio. Its ferroelasticity with moderate switching barrier is promising for applications in shape memory devices.
\end{abstract}

DOI: 10.1103/PhysRevApplied.14.044015

\section{INTRODUCTION}

Black phosphorene ( $\alpha$-phosphorene) has attracted tremendous interest owing to its potential applications in numerous important fields [1,2]. For instance, its moderate direct band gap is promising for use in optical devices, such as photodetectors [3] and solar cells [4,5]. It is an out-of-plane auxetic material [with a negative Poisson's ratio (NPR) of -0.027] [6,7]. In addition, it combines high-mobility charge carriers with transport anisotropy [8,9], which can be switched by a ferroelastic transition [10]. Going beyond black phosphorene, Guan and co-workers have proposed $\beta$-, $\gamma-$, and $\delta$-phosphorene as allotropes [11]. While both $\beta$ - and $\gamma$-phosphorene are indirect-band-gap semiconductors, a direct band gap of $0.66 \mathrm{eV}$ (predicted by the Heyd-Scuseria-Ernzerhof functional) renders $\delta$-phosphorene suitable for optical devices operating in the infrared region but not for solar cell applications, which require a band gap of approximately $1 \mathrm{eV}$ to achieve a high power conversion efficiency (PCE). The NPR of $\delta$-phosphorene ( -0.267 [12]) exceeds that of most two-dimensional (2D) auxetic materials, whereas ferroelasticity is hindered by a low ratio of the lattice constants $(5.56 / 5.46 \approx 1.02[11])$.

As group IV-VI binary 2D materials are isoelectronic to group V 2D materials, it is of great interest to know whether they share properties with the phosphorene allotropes. The group IV-VI binary 2D material SnS, which resembles the structure of $\alpha$-phosphorene, has been put forward for thermoelectric [13], piezoelectric [14], and ferroelectronic [15] applications. In the present work, we predict the properties of a $\delta$-phosphorene-like group IV-VI binary 2D material, $\delta$-CS, using first-principles calculations. We demonstrate that it combines excellent

*udo.schwingenschlogl@kaust.edu.sa mechanical, electronic, and optical characteristics. It has a moderate direct band gap with strong absorption of solar radiation, is auxetic, and has switchable directional electric and optical properties due to its ferroelasticity. This outstanding multifunctionality has potential applications in 2D nanoelectronic and nano-optical devices.

\section{METHODOLOGY}

First-principles calculations are performed using density functional theory (Vienna ab initio simulation package [16] using the projector-augmented wave method with a plane-wave cutoff energy of $500 \mathrm{eV}$ ). Both the PerdewBurke-Ernzerhof (crystal structure) and Heyd-ScuseriaErnzerhof [17] (band structure, with the standard screening parameter of $0.2 \AA^{-1}$ and the standard mixing parameter of 0.25 ) exchange-correlation functionals are adopted. Taking into account electron-electron correlations and electron-hole interactions [18,19], the optical absorption spectrum is obtained by combining the $G_{0} W_{0}$ approach with the Bethe-Salpeter equation (where the plane-wave cutoff energy is set to $500 \mathrm{eV}$ ) [20,21]. A 20- $\AA$-thick vacuum layer is used to construct a $2 \mathrm{D}$ model with periodic boundary conditions. The Brillouin zone is sampled on an $8 \times 8 \times 1 \quad \Gamma$-centered $k$-mesh and the convergence criteria of the energy and force are set to $10^{-8} \mathrm{eV}$ and $10^{-4} \mathrm{eV} / \AA$, respectively. Phonon spectra are calculated by density functional perturbation theory (using the PHONOPY package). Ab initio molecular dynamics (AIMD) simulations are carried out using a $4 \times 4$ supercell (128 atoms; Perdew-Burke-Ernzerhof exchange-correlation functional) and an NVT ensemble with the temperature controlled by the Nosé-Hoover method [22]. At each temperature, the AIMD simulation is conducted for 5 ps with a time step of $1 \mathrm{fs}$. The path and energy barrier of the ferroelastic 
transition are obtained using the solid-state nudged elastic band method [23].

\section{RESULTS AND DISCUSSION}

The anisotropic atomic structure of $\delta$-CS is shown in Fig. 1(a). It has a rectangular unit cell with space group $P c a 2_{1}$ containing $4 \mathrm{C}$ and $4 \mathrm{~S}$ atoms. We obtain lattice constants of $a=4.51 \AA$ and $b=4.97 \AA(b / a \sim 1.1)$. The cohesive energy, $\left(4 E_{\mathrm{C}}+4 E_{\mathrm{S}}-E_{\delta-\mathrm{CS}}\right) / 8$, where $E_{\mathrm{C}}, E_{\mathrm{S}}$, and $E_{\delta-C S}$ denote the total energies of a $\mathrm{C}$ atom, an $\mathrm{S}$ atom, and the unit cell, respectively, is as high as $4.12 \mathrm{eV} /$ atom, i.e., higher than that of silicene (3.91 eV/atom), phosphorene (3.48 eV/atom), and germanene (3.24 eV/atom), demonstrating energetic stability and feasibility of synthesis. This cohesive energy is also higher than that of the competing space groups $P 3 \mathrm{ml}(3.98 \mathrm{eV} /$ atom) and $P \overline{6} \mathrm{ml}$ (3.48 eV/atom). The phonon dispersion in Fig. 1(b) (3 acoustic branches, 21 optical branches) shows no imaginary frequency and a maximum frequency as high as $849 \mathrm{~cm}^{-1}$, suggesting dynamic stability. According to Fig. 1(c), no phase transition, no bond breaking, and no significant structural distortion is encountered in an AIMD simulation at $300 \mathrm{~K}$, which verifies thermal stability. The structure remains stable at $400 \mathrm{~K}$ but not at $500 \mathrm{~K}$, suggesting that the melting point lies between 400 and $500 \mathrm{~K}$ (Figures S1 and S2 in the Supplemental Material [24]). Notably, $\delta$-CS is also stable under ambient conditions, as $\mathrm{O}_{2}$ molecules interact only by physisorption. Overall, our results indicate that the most promising approach to experimental synthesis of $\delta$-CS is low-temperature epitaxial growth.

The band structure in Fig. 2(a) shows that $\delta$-CS is a semiconductor with a direct band gap of $1.28 \mathrm{eV}$. Both the valence band maximum (VBM) and conduction band minimum (CBM) are located at the center of the Brillouin zone ( $\Gamma$ point). According to the density of states in Fig. 2(a) and the charge distributions in Figs. 2(b) and 2(c), the VBM is dominated by $\mathrm{C} p_{z}$ and $\mathrm{S} p_{z}$ states and the CBM by $\mathrm{C} p_{z}$, and $\mathrm{S} p_{x, y}$ states. Calculation of the electron and hole effective masses, $\hbar^{2}\left[\partial^{2} \varepsilon(k) / \partial k^{2}\right]^{-1}$, results in a distinct direction dependence (anisotropic electrical conductance), see Fig. 2(d), with values of $0.55(0.66) m_{e}$ for the electrons and $0.48(1.05) m_{e}$ for the holes in the $x(y)$ direction. As the narrow band gap of $\delta$-CS is promising for solar energy harvesting, we explore the optical absorption by calculating the complex dielectric function $\varepsilon(\omega)=\varepsilon_{1}(\omega)+i \varepsilon_{2}(\omega)$. The absorption spectrum in Fig. 2(e) suggests that $\delta$-CS strongly absorbs $x$-polarized light at around $1 \mathrm{eV}$ but is transparent to $y$-polarized light in this region. This property is a consequence of the anisotropic crystal structure and has potential applications in near-infrared linear polarizers. The fact that the optical absorption of $\delta$-CS covers the entire solar spectrum is important for photovoltaics. We note that the obtained values resemble those of monolayer $\mathrm{MoS}_{2}$, for which a solar cell with more than $5 \%$ power conversion efficiency has been demonstrated experimentally in Ref. [25].

Because of the semiconducting nature of $\delta$-CS with a moderate direct band gap and excellent absorption of solar radiation, we evaluate the material's performance in 2D heterostructure solar cells. As the second component we select the transition metal dichalcogenides (TMDs) $\mathrm{MoS}_{2}$, $\mathrm{MoSe}_{2}$, and $\mathrm{MoTe}_{2}$, which also combine a moderate direct band gap with good optical absorption $[26,27]$ and achieve a favorable performance in photovoltaic devices [28,29]. A summary of the lattice constants and band gaps of the TMDs is given in Table S1 in the Supplemental Material [24] and their band structures are shown in Fig. S3 in the Supplemental Material [24]. Figure 3(a) summarizes the band alignments in $\delta$-CS and the TMDs. The valence (conduction) band edge of $\delta$-CS, $\mathrm{MoS}_{2}, \mathrm{MoSe}_{2}$, and $\mathrm{MoTe}_{2}$ is found at an energy of $-4.82(-3.55),-6.38(-4.23)$, $-5.75(-3.86)$, and $-5.19(-3.68) \mathrm{eV}$ with respect to the (a)

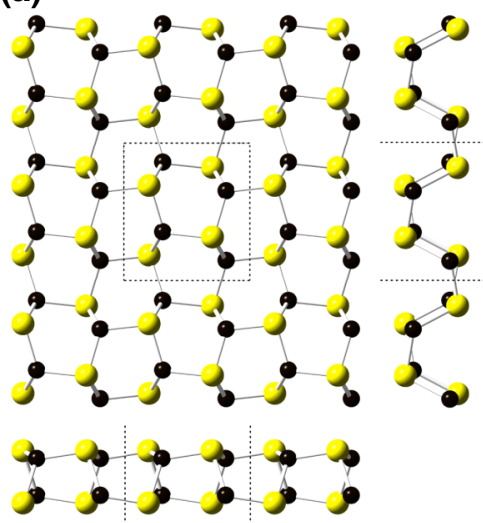

(b)

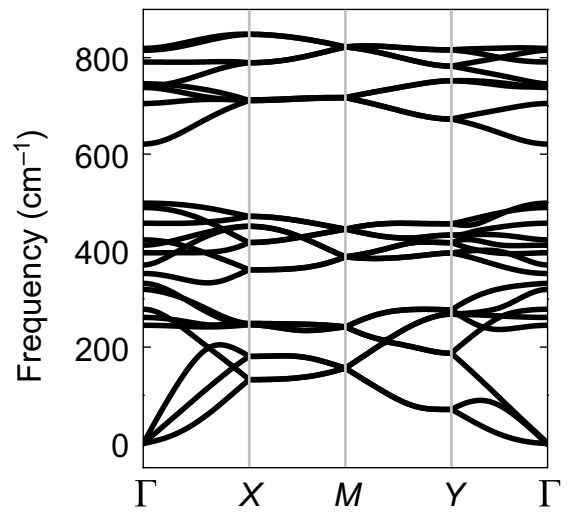

(c)

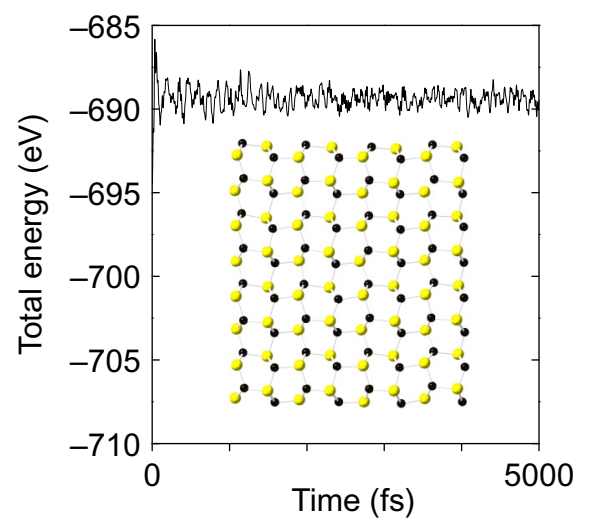

FIG. 1. (a) Atomic structure, (b) phonon dispersion, and (c) total potential energy fluctuation during an AIMD simulation at $300 \mathrm{~K}$ (inset: atomic structure after $5 \mathrm{ps)}$ of $\delta$-CS. 

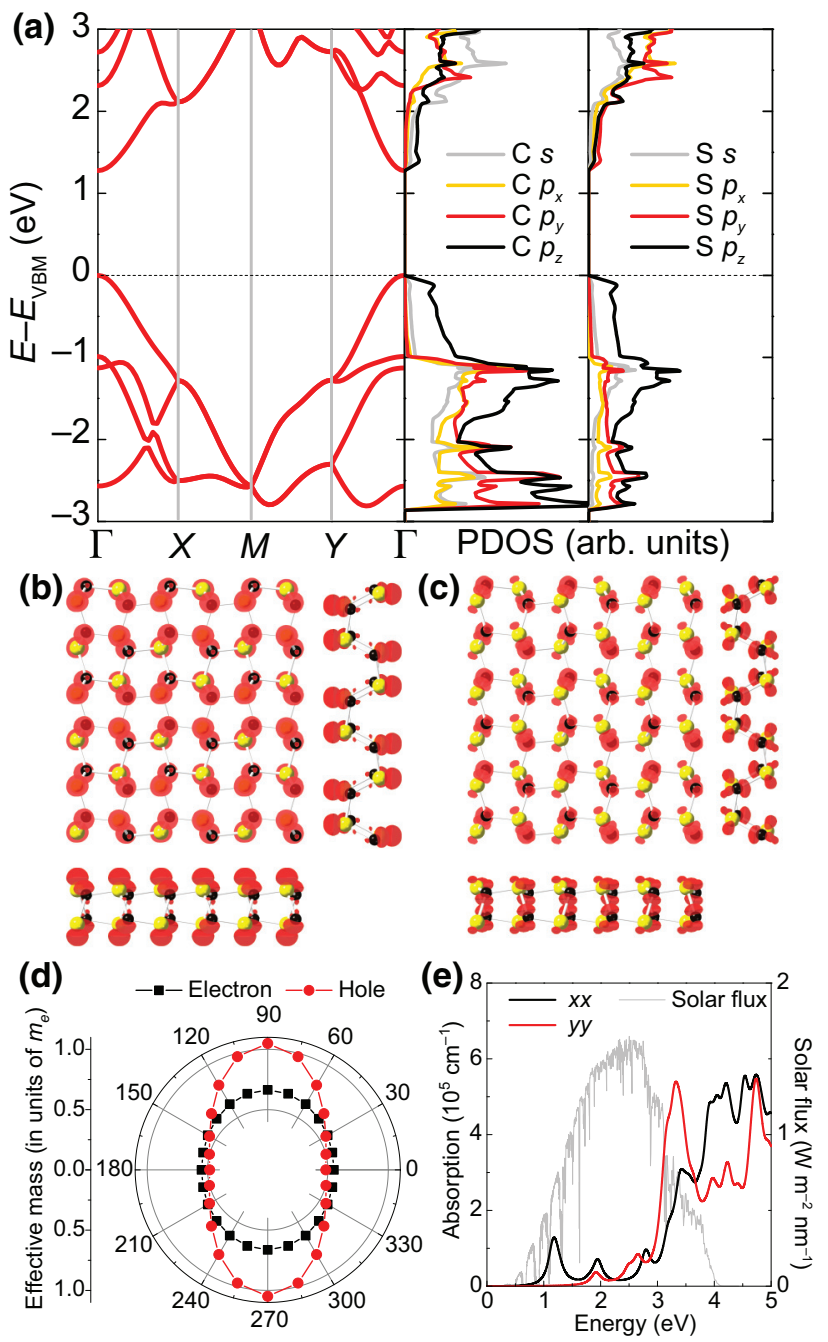

FIG. 2. (a) Band structure and partial densities of states (PDOS) calculated by the Heyd-Scuseria-Ernzerhof functional (VBM set to zero energy and indicated by a black dashed line), charge distribution (isovalue $0.1 \mathrm{e}^{-3}$ ) at the (b) VBM and (c) CBM, (d) direction dependences of the electron and hole effective masses, and (e) absorption spectrum of $\delta$-CS.

vacuum level, respectively. Thus, we obtain a type-II band alignment when $\delta$-CS forms a heterostructure with any of the three TMDs. As the electron affinity (energy difference between the CBM and vacuum level) is smaller for $\delta$-CS than for the TMDs, $\delta$-CS acts as an electron donor. The conduction band offsets of $\delta$-CS to $\mathrm{MoS}_{2}, \mathrm{MoSe}_{2}$, and $\mathrm{MoTe}_{2}$ turn out to be $0.68,0.31$, and $0.13 \mathrm{eV}$, respectively. [30]

The maximum PCE of a solar cell can be estimated as

$$
\mathrm{PCE}_{\max }=\frac{\beta_{\mathrm{FF}} V_{\mathrm{OC}} J_{\mathrm{SC}}}{P_{\text {solar }}}
$$

where $\beta_{\mathrm{FF}}=0.65$ is the fill factor, $V_{\mathrm{OC}}$ is the open circuit voltage, $J_{\mathrm{SC}}$ is the short circuit current in the limit of $100 \%$ external quantum efficiency, and $P_{\text {solar }}$ is the incident solar (a)
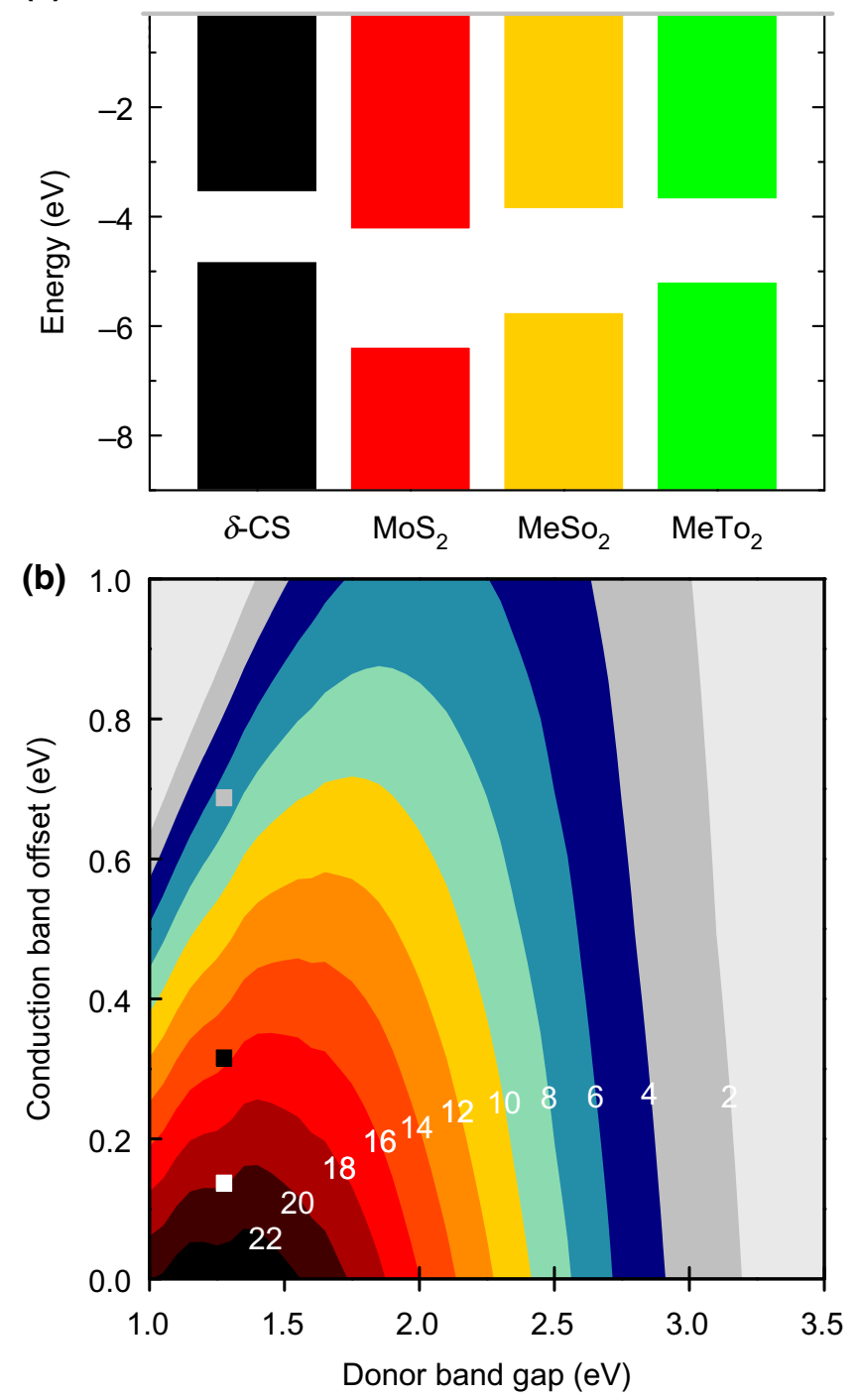

FIG. 3. (a) Band alignment in $\delta$-CS and the TMDs. (b) $\mathrm{PCE}_{\max }$ (in \%) [Eq. (1)]. Gray, black, and white squares mark $\delta$-CS/MoS $2, \delta$-CS/MoSe 2 , and $\delta$-CS/MoTe 2 , respectively.

energy per unit area. We can write

$$
\begin{aligned}
& V_{\mathrm{OC}}=\frac{1}{e}\left(E_{g}^{d}-\Delta E_{c}-0.3\right), \\
& J_{\mathrm{SC}}=e \int_{E_{g}}^{\infty} \frac{P(\hbar \omega)}{\hbar \omega} d(\hbar \omega), \\
& P_{\text {solar }}=\int_{0}^{\infty} P(\hbar \omega) d(\hbar \omega),
\end{aligned}
$$

where $E_{g}^{d}$ is the optical gap of the donor, which we approximate to be the band gap of $1.28 \mathrm{eV}, \Delta E_{c}$ is the 
conduction band offset between the donor and acceptor, $\hbar \omega$ is the photon energy, and $P(\hbar \omega)$ is the AM1.5 solar energy flux. We obtain $\mathrm{PCE}_{\max }=7.0 \%$ for $\delta-\mathrm{CS} / \mathrm{MoS}_{2}$, $15.8 \%$ for $\delta$-CS/MoSe 2 , and $20.1 \%$ for $\delta$-CS/MoTe 2 , see Fig. 3(b). These values fall short of state-of-theart solar cells (silicon: 27.6\%, GaAs: 30.5\% [31]) but are comparable with 2D fullerene-based $\left(\mathrm{PCE}_{\max }=20 \%\right)$ [32], phosphorene/TMD ( $\left.\mathrm{PCE}_{\max }=12 \%\right)$ [5], and bilayerphosphorene $/ \mathrm{MoS}_{2}\left(\mathrm{PCE}_{\max }=18 \%\right)$ [33] solar cells.

The mechanical properties of $\delta$-CS are investigated by calculating the elastic constants. For graphene we obtain $C_{11}=C_{22}=346 \mathrm{~N} / \mathrm{m}$ and $C_{12}=C_{21}=66 \mathrm{~N} / \mathrm{m}$, which is consistent with previous work [34] and demonstrates the reliability of the computational method. For $\delta$-CS the elastic constants turn out to be $C_{11}=147 \mathrm{~N} / \mathrm{m}, C_{22}=41 \mathrm{~N} / \mathrm{m}$, $C_{12}=C_{21}=-5 \mathrm{~N} / \mathrm{m}$, and $C_{66}=42 \mathrm{~N} / \mathrm{m}$, satisfying the Born criteria $\mathrm{C}_{11} C_{22}-C_{12}^{2}>0$ and $C_{66}>0$, and therefore demonstrating mechanical stability. The flexibility of a material can be described by Young's modulus $\left[E_{x}=\left(C_{11} C_{22}-C_{12} C_{21}\right) / C_{22}, E_{y}=\left(C_{11} C_{22}-C_{12} C_{21}\right) / C_{11}\right]$ and Poisson's ratio $\left(v_{x}=C_{21} / C_{22}, v_{y}=C_{12} / C_{11}\right)$. We obtain for $\delta$-CS values of $E_{x}=146 \mathrm{~N} / \mathrm{m}$ and $E_{y}=41 \mathrm{~N} / \mathrm{m}$ (more than three times higher in the $x$ than in the $y$ direction), suggesting large mechanical anisotropy. The fact that $\delta$-CS is much more flexible than graphene $\left(E_{x}=E_{y}=333 \mathrm{~N} / \mathrm{m}\right)$ opens the possibility of a ferroelastic transition under external strain (as discussed later). The calculated values of Poisson's ratio, $v_{x}=-0.12$ and $v_{y}=-0.03$, confirm a large mechanical anisotropy and show that $\delta$-CS is an auxetic material.

The response of the lattice constants of $\delta$-CS to uniaxial strain is addressed in Fig. 4(a). We observe that the lattice constant in the $x(y)$ direction increases for increasing strain in the $y(x)$ direction, in agreement with the NPR. In contrast to the out-of-plane NPR of phosphorene [7], borophane [35], and titanium mononitride [36], the NPR of $\delta$-CS is of in-plane type. The values of the inplane NPR of $\delta$-CS $\left(v_{x}=-0.12, v_{y}=-0.03\right)$ exceed those of penta-graphene $(-0.068)$ [37] and borophene $(-0.02$ and -0.04) [38], are comparable with those of silicon oxide (from -0.022 to -0.123 ) [39], and fall short of those of strained graphene $(-0.333)$ [40], $\delta$-phosphorene $(-0.158$ and -0.267$)[12]$ and $\mathrm{Be}_{5} \mathrm{C}_{2}(-0.041$ and -0.16$)$ [41]. A moderate in-plane NPR is promising for sensors and acoustic isolators, for example. From the strain-stress curves in Fig. 4(b) we calculate fracture strengths of $11.7 \mathrm{~N} / \mathrm{m}$ in the $x$ direction and $3.9 \mathrm{~N} / \mathrm{m}$ in the $y$ direction. The obtained fracture strains of $13 \%$ in the $x$ direction and $14 \%$ in the $y$ direction are relatively small, which must be carefully considered in future applications of $\delta$-CS to avoid mechanical failure. It is confirmed that the phonon spectra show no imaginary frequencies up to the determined fracture strains.

As in a ferroelastic material switching between two or more equally stable states is possible by means of external stress, the material must be anisotropic. Two equally stable states $\left(S\right.$ and $\left.S^{\prime}\right)$ of anisotropic $\delta$-CS with different orientations $\left(90^{\circ}\right.$ rotation) are shown as insets in Fig. 5. We use the solid-state nudged elastic band method to determine the transition path and energy barrier between them. As shown in Fig. 5, the initial state $S$ converts into the final state $S^{\prime}$ through a paraelastic transition state $T$ with a square lattice $(a=b=4.70 \AA)$. According to the calculated phonon dispersion (Fig. S4 in the Supplemental Material [24]), the transition state is unstable, i.e., it will spontaneously transform into the $S$ or $S^{\prime}$ state. Therefore, $\delta$-CS is a 2D ferroelastic material.

The energy barrier of the ferroelastic transition must be sufficiently small to facilitate switching, but must be sufficiently large to guarantee stability against thermal fluctuations and, therefore, reliable device operation. We obtain a value of $36 \mathrm{meV} /$ atom $(291 \mathrm{meV} /$ unit cell, see Fig. 5), which is smaller than that of $\mathrm{BP}_{5}(320 \mathrm{meV} /$ atom $)$ [42], $\alpha$-phosphorene (200 meV/atom) [10], and borophane (100 meV/atom) [35], comparable with that of tetragonal yttrium nitride (33 meV/atom) [43], and larger than that of group IV-VI binary compounds (1.2-22.6 meV/atom) [44] and titanium mononitride ( $0.8 \mathrm{meV} /$ atom $)$ [36]. The moderate energy barrier of $\delta$-CS not only enables fast switching, but also prevents spontaneous domain wall motion.
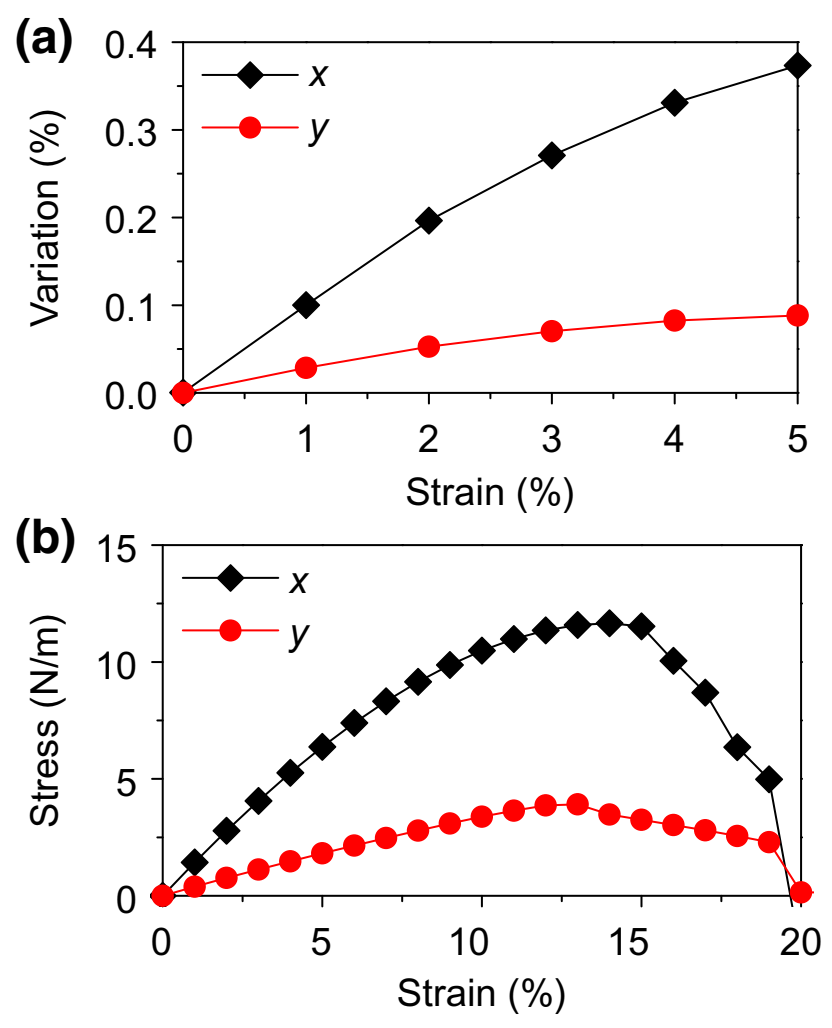

FIG. 4. (a) Response of the lattice constant in the $x(y)$ direction to uniaxial strain in the $y(x)$ direction. (b) Strain-stress curves for the $x$ and $y$ directions. 


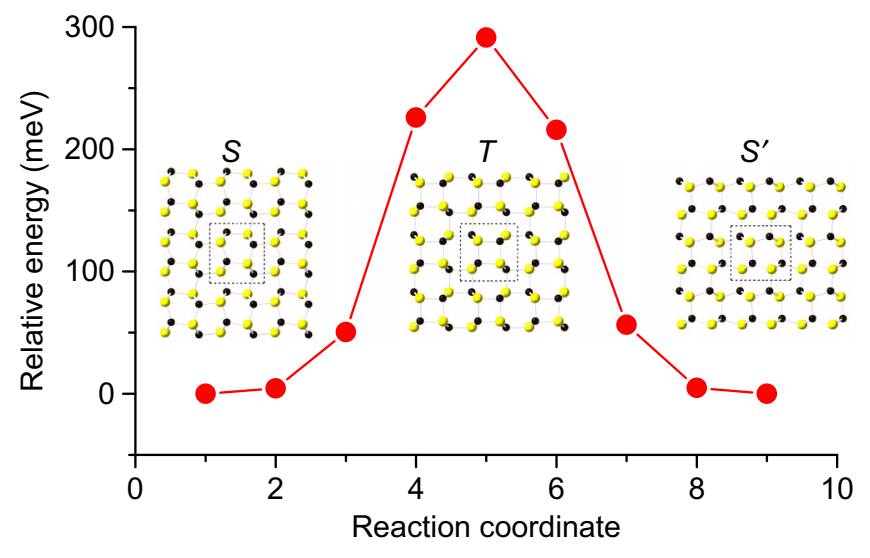

FIG. 5. Energy profile of the ferroelastic switching in $\delta$-CS between equally stable states $S$ and $S^{\prime}$ with different orientations through a paraelastic state $T$.

The external strain required for triggering the ferroelastic transition is derived from the Green-Lagrange strain tensor [45], resulting in values of only $5.9 \%$ for the $x$ direction and $-4.0 \%$ for the $y$ direction. Combined with a moderate energy barrier, such low threshold strains are promising for shape memory devices.

\section{CONCLUSION}

In conclusion, we propose a highly anisotropic 2D material, $\delta$-CS, for which we demonstrate stability under ambient conditions by considering the cohesive energy, the phonon spectrum, AIMD simulations, and the elastic constants. We show that the material is a direct-bandgap $(1.28 \mathrm{eV})$ semiconductor with strong absorption of solar radiation, rendering it promising for utilization in photovoltaic devices. According to the calculated band alignments, $\delta$-CS acts as an electron donor in contact with $\mathrm{MoS}_{2}, \mathrm{MoSe}_{2}$, and $\mathrm{MoTe}_{2}$. We predict excellent PCEs of $7.0 \%, 15.8 \%$, and $20.1 \%$ for $\delta-\mathrm{CS} / \mathrm{MoS}_{2}, \delta$ $\mathrm{CS} / \mathrm{MoSe}_{2}$, and $\delta$-CS/MoTe ${ }_{2}$ heterostructure solar cells. Our results also show that $\delta$-CS is auxetic and ferroelastic. The in-plane NPR turns out to be superior to numerous 2D materials; for instance, it outperforms borophene by a factor of almost 3 . The ferroelastic transition of $\delta$-CS combines a moderate energy barrier ( $36 \mathrm{meV} / \mathrm{atom})$ with low-threshold strains $(5.9 \%$ in the $x$ direction, $-4.0 \%$ in the $y$ direction), as desired for shape memory devices.

\section{ACKNOWLEDGMENTS}

The research reported in this publication was supported by funding from King Abdullah University of Science and Technology (KAUST). We thank Dr. Huabing Shu and Mr. Ziang Zhang for assistance with PYTHON programming.
[1] H. Liu, A. T. Neal, Z. Zhu, Z. Luo, X. F. Xu, D. Tomanek, and P. D. Ye, Phosphorene: An unexplored 2D semiconductor with a high hole mobility, ACS Nano 8, 4033 (2014).

[2] A. Carvalho, M. Wang, X. Zhu, A. S. Rodin, H. Su, and A. H. Castro Neto, Phosphorene: From theory to applications, Nat. Rev. Mater. 1, 16061 (2016).

[3] N. Youngblood, C. Chen, S. J. Koester, and M. Li, Waveguide-integrated black phosphorus photodetector with high responsivity and low dark current, Nat. Photonics 9, 247 (2015).

[4] L. Zhou, J. Zhang, Z. Zhuo, L. Kou, W. Ma, B. Shao, A. $\mathrm{Du}, \mathrm{S}$. Meng, and T. Frauenheim, Novel excitonic solar cells in phosphorene- $\mathrm{TiO}_{2}$ heterostructures with extraordinary charge separation efficiency, J. Phys. Chem. Lett. 7, 1880 (2016).

[5] V. Dheivanayagam, J. Linghu, C. Zhang, Y. P. Feng, and L. Shen, Heterostructures of phosphorene and transition metal dichalcogenides for excitonic solar cells: A first-principles study, Appl. Phys. Lett. 108, 122105 (2016).

[6] J.-W. Jiang and H. S. Park, Negative Poisson's ratio in single-layer black phosphorus, Nat. Commun. 5, 4727 (2014).

[7] Y. Du, J. Maassen, W. Wu, Z. Luo, X. Xu, and P. D. Ye, Auxetic black phosphorus: A 2D material with negative Poisson's ratio, Nano Lett. 16, 6701 (2016).

[8] J. Qiao, X. Kong, Z.-X. Hu, F. Yang, and W. Ji, Highmobility transport anisotropy and linear dichroism in fewlayer black phosphorus, Nat. Commun. 5, 4475 (2014).

[9] R. Fei and L. Yang, Strain-engineering the anisotropic electrical conductance of few-layer black phosphorus, Nano Lett. 14, 2884 (2014).

[10] M. Wu and X. C. Zeng, Intrinsic ferroelasticity and/or multiferroicity in two-dimensional phosphorene and phosphorene analogues, Nano Lett. 16, 3236 (2016).

[11] J. Guan, Z. Zhu, and D. Tomanek, Phase Coexistence and Metal-Insulator Transition in Few-Layer Phosphorene: A Computational Study, Phys. Rev. Lett. 113, 046804 (2014).

[12] H. Wang, X. Li, P. Li, and J. Yang, $\delta$-phosphorene: A two dimensional material with a highly negative Poisson's ratio, Nanoscale 9, 850 (2017).

[13] Q. Tan, L.-D. Zhao, J.-F. Li, C.-F. Wu, T.-R. Wei, Z.-B. Xing, and M. G. Kanatzidis, Thermoelectrics with earth abundant elements: Low thermal conductivity and high thermopower in doped SnS, J. Mater. Chem. A 2, 17302 (2014).

[14] R. Fei, W. Li, J. Li, and L. Yang, Giant piezoelectricity of monolayer group IV monochalcogenides: $\mathrm{SnSe}, \mathrm{SnS}, \mathrm{GeSe}$, and GeS, Appl. Phys. Lett. 107, 173104 (2015).

[15] Y. Bao, P. Song, Y. Liu, Z. Chen, M. Zhu, I. Abdelwahab, J. Su, W. Fu, X. Chi, W. Yu, W. Liu, X. Zhao, Q.-H. Xu, M. Yang, and K. P. Loh, Gate-tunable in-plane ferroelectricity in few-layer SnS, Nano Lett. 19, 5109 (2019).

[16] G. Kresse and D. Joubert, From ultrasoft pseudopotentials to the projector augmented-wave method, Phys. Rev. B 59, 1758 (1999).

[17] J. Heyd, G. E. Scuseria, and M. Ernzerhof, Hybrid functionals based on a screened Coulomb potential, J. Chem. Phys. 118, 8207 (2003). 
[18] G. Onida, L. Reining, and A. Rubio, Electronic excitations: Density-functional versus many-body Green'sfunction approaches, Rev. Mod. Phys. 74, 601 (2002).

[19] L. Wirtz, A. Marini, and A. Rubio, Excitons in Boron Nitride Nanotubes: Dimensionality Effects, Phys. Rev. Lett. 96, 126104 (2006).

[20] S. Albrecht, L. Reining, R. Del Sole, and G. Onida, Ab Initio Calculation of Excitonic Effects in the Optical Spectra of Semiconductors, Phys. Rev. Lett. 80, 4510 (1998).

[21] M. Rohlfing and S. G. Louie, Electron-Hole Excitations in Semiconductors and Insulators, Phys. Rev. Lett. 81, 2312 (1998).

[22] G. J. Martyna, M. L. Klein, and M. Tuckerman, NoséHoover chains: The canonical ensemble via continuous dynamics, J. Chem. Phys. 97, 2635 (1992).

[23] D. Sheppard, P. Xiao, W. Chemelewski, D. D. Johnson, and G. Henkelman, A generalized solid-state nudged elastic band method, J. Chem. Phys. 136, 074103 (2012).

[24] See Supplemental Material at http://link.aps.org/supple mental/10.1103/PhysRevApplied.14.044015 for AIMD simulations of $\delta$-CS at 400 and $500 \mathrm{~K}$, lattice constants and band gaps of TMDs, band structures of TMDs, and phonon dispersion of the paraelastic state of $\delta$-CS.

[25] M.-L. Tsai, S.-H. Su, J.-K. Chang, D.-S. Tsai, C.-H. Chen, C.-I. Wu, L.-J. Li, L.-J. Chen, and J.-H. He, Monolayer $\mathrm{MoS}_{2}$ heterojunction solar cells, ACS Nano 8, 8317 (2014).

[26] M. Palummo, M. Bernardi, and J. C. Grossman, Exciton radiative lifetimes in two-dimensional transition metal dichalcogenides, Nano Lett. 15, 2794 (2015).

[27] J. Yang, T. Lü, Y. W. Myint, J. Pei, D. Macdonald, J.-C. Zheng, and Y. Lu, Robust excitons and trions in monolayer $\mathrm{MoTe}_{2}$, ACS Nano 9, 6603 (2015).

[28] M. Bernardi, M. Palummo, and J. C. Grossman, Extraordinary sunlight absorption and one nanometer thick photovoltaics using two-dimensional monolayer materials, Nano Lett. 13, 3664 (2013).

[29] S. Memaran, N. R. Pradhan, Z. Lu, D. Rhodes, J. Ludwig, Q. Zhou, O. Ogunsolu, P. M. Ajayan, D. Smirnov, A. I. Fernández-Domínguez, F. J. García-Vidal, and L. Balicas, Pronounced photovoltaic response from multilayered transition-metal dichalcogenides PN-junctions, Nano Lett. 15, 7532 (2015).

[30] M. C. Scharber, D. Mühlbacher, M. Koppe, P. Denk, C. Waldauf, A. J. Heeger, and C. J. Brabec, Design rules for donors in bulk-heterojunction solar cells - towards $10 \%$ energy-conversion efficiency, Adv. Mater. 18, 789 (2006).

[31] M. A. Green, Y. Hishikawa, E. D. Dunlop, D. H. Levi, J. HohlEbinger, M. Yoshita, and A. W. Y. Ho-Baillie, Solar cell efficiency tables (Version 53), Prog. Photovoltaics 27, 3 (2019).
[32] M. Bernardi, M. Palummo, and J. C. Grossman, Semiconducting monolayer materials as a tunable platform for excitonic solar cells, ACS Nano 6, 10082 (2012).

[33] J. Dai and X. C. Zeng, Bilayer phosphorene: Effect of stacking order on bandgap and Its potential applications in thin-film solar cells, J. Phys. Chem. Lett. 5, 1289 (2014).

[34] B. Wang, Q. Wu, Y. Zhang, L. Ma, and J. Wang, Auxetic $\mathrm{B}_{4} \mathrm{~N}$ monolayer: A promising 2D material with in-plane negative Poisson's ratio and large anisotropic mechanics, ACS Appl. Mater. Interfaces 11, 33231 (2019).

[35] L. Kou, Y. Ma, C. Tang, Z. Sun, A. Du, and C. Chen, Auxetic and ferroelastic borophane: A novel 2D material with negative Possion's ratio and switchable dirac transport channels, Nano Lett. 16, 7910 (2016).

[36] L. Zhou, Z. Zhuo, L. Kou, A. Du, and S. Tretiak, Computational dissection of two-dimensional rectangular titanium mononitride tin: Auxetics and promises for photocatalysis, Nano Lett. 17, 4466 (2017).

[37] S. Zhang, J. Zhou, Q. Wang, X. Chen, Y. Kawazoe, and P. Jena, Penta-graphene: A new carbon allotrope, Proc. Natl. Acad. Sci. U. S. A. 112, 2372 (2015).

[38] A. J. Mannix, X.-F. Zhou, B. Kiraly, J. D. Wood, D. Alducin, B. D. Myers, X. Liu, B. L. Fisher, U. Santiago, and J. R. Guest, Synthesis of borophenes: Anisotropic, two-dimensional boron polymorphs, Science 350, 1513 (2015).

[39] Z. Gao, X. Dong, N. Li, and J. Ren, Novel two-dimensional silicon dioxide with in-plane negative Poisson's ratio, Nano Lett. 17, 772 (2017).

[40] J.-W. Jiang, T. Chang, X. Guo, and H. S. Park, Intrinsic negative Poisson's ratio for single-layer graphene, Nano Lett. 16, 5286 (2016).

[41] Y. Wang, F. Li, Y. Li, and Z. Chen, Semi-metallic $\mathrm{Be}_{5} \mathrm{C}_{2}$ monolayer global minimum with quasi-planar pentacoordinate carbons and negative Poisson's ratio, Nat. Commun. 7, 11488 (2016).

[42] H. Wang, X. Li, J. Sun, Z. Liu, and J. Yang, BP 5 monolayer with multiferroicity and negative Poisson's ratio: A prediction by global optimization method, 2D Mater. 4, 045020 (2017)

[43] B. Xu, H. Xiang, J. Yin, Y. Xia, and Z. Liu, A Twodimensional tetragonal yttrium nitride monolayer: A ferroelastic semiconductor with switchable anisotropic properties, Nanoscale 10, 215 (2018).

[44] H. Wang and X. Qian, Two-dimensional multiferroics in monolayer group IV monochalcogenides, 2D Mater. 4, 015042 (2017).

[45] W. Li and J. Li, Ferroelasticity and domain physics in twodimensional transition metal dichalcogenide monolayers, Nat. Commun. 7, 10843 (2016). 